\title{
Division of Maternal-Fetal Medicine
}

The Division of Maternal-Fetal Medicine presently includes nine board certified maternal-fetal specialists. They are: Kimberly K. Leslie, MD (Department Head), Jerome Yankowitz, MD (Division Director), Jennifer R. Niebyl, MD, Asha Rijhsinghani, MD, Janet I. Andrews, MD, Stephen K. Hunter, MD, PhD., Kristi Borowski, MD, Mark Santillan, MD, and Andrea Greiner, MD. Dr. Roger A. Williamson, double boarded in Genetics and Obstetrics, is also a member of the Division. Drs. Yankowitz and Borowski are also board certified in clinical genetics.

Several hundred of the deliveries resulted from maternal transports. Roughly $70 \%$ of the patients delivered at the University of lowa have clear cut medical, surgical, or obstetrical complications of pregnancy. There are almost 500 antepartum admissions per year. If demographic factors such as marital status, age, and socioeconomic status are included in the definition of high risk, at least $80 \%$ of the population is easily classified as high risk.

The patients are cared for in a series of clinics. The High Risk Obstetric Clinic meets three days a week. Many insulin dependent and gestationally diabetic women receive care through the High Risk Service annually. In addition, there is a representative sample of nearly all other complications of pregnancy.

Genetic care in the state is regionalized, and when indicated, the patient is referred to the University. The section of Obstetrical Genetics consists of Dr. Roger A. Williamson (Director), Dr. Kristi Borowski, and Dr. Jerome Yankowitz. A statewide maternal serum-screening program is regionalized by the central State Hygienic Laboratory by statute. Dr. Williamson is the physician coordinator for the program. Triple hormone screening began in June 1990, and some 14,000 women are screened each year. The Genetics Section encounters approximately 1,300 patients annually. Of these, about 500 undergo amniocentesis or CVS. During six half-day sessions per week, evaluation and counseling of patients of known or presumed risk for genetic disorders is carried out. The Genetics Clinic is a part of the Department of Obstetrics and Gynecology and it enjoys a close working relationship with the Division of Medical Genetics in the Department of Pediatrics.

The University of lowa has a wellestablished multidisciplinary Fetal Diagnosis and Treatment Unit under the direction of Dr. Yankowitz. Level II scans, anomaly consultations, and interventional fetal procedures are carried out in this unit. Many cordocenteses and intravascular transfusions are performed each year. The major interest of the team is the development of fetal medicine. The Obstetrics and Gynecology Ultrasound Program is certified by the American Institute of Ultrasound in Medicine (AIUM).

The division is approved for one maternalfetal medicine fellow each year and a combined MFM-clinical genetics fellow every other year. Only 10 programs have such combined programs nationwide.

Jerome Yankowitz, MD

Division Director, Maternal-Fetal Medicine 